\title{
Thymic Carcinoid Tumor
}

National Cancer Institute

\section{Source}

National Cancer Institute. Thymic Carcinoid Tumor. NCI Thesaurus. Code C6430.

A primary thymic neuroendocrine tumor, often associated with Cushing syndrome. Two

morphologic subtypes are recognized: typical and atypical carcinoid tumors. Atypical

carcinoid tumors have a more aggressive clinical course. 